Cesar Winnicki ${ }^{\mathrm{a}}$
Bernd Orzessek

\section{Komplementärmedizinische \\ Ansätze in der Behandlung}

Die bedeutendste Option in der komplementärmedizinischen Behandlung von Patienten mit chronischentzündlichen Darmerkrankungen (CED) stellt die Behandlung mit natürlichen Darmflorabakterien dar. Die sogenannte probiotische Therapie gehört seit den 50er-Jahren des 20. Jahrhunderts zu den Hauptdomänen der Komplementärmedizin. Die beachtlichen Erfolge dieser natürlichen, nebenwirkungsfreien Behandlung erweckte nach einigen Jahrzehnten auch das Interesse der konventionellen Medizin - mit anschliessendem Nachweis der Wirksamkeit durch klinische Studien. Die Bedeutung der intakten Darmflora für die Gesundheit des Menschen wurde vor fast 100 Jahren zum ersten Mal bewusst durch den russischen Mikrobiologen Elias Mietchnikov (1908 Nobelpreis für Medizin) beschrieben. Die Wechselwirkung zwischen der Darmflora und der Gesundheit drückte er mit folgenden Worten aus:

«Die meist naturwidrige Lebensweise des Menschen führt zu einer aussergewöhnlichen, lebhaften Entwicklung schädlicher Bakterien im Dünndarm und dadurch zu chronischer Selbstvergiftung, Krankheiten aller Art, Siechtum, frühzeitigem Altern und vorzeitigem Tod.»

Mietchnikov führte die auffallende Langlebigkeit der südeuropäischen Bevölkerung auf den hohen Joghurt-

aeskulap-Klinik, Brunnen, Schweiz

\title{
Chronisch-entzündliche Darmerkrankungen aus der Sicht der Komplementärmedizin - Teil 2
}

konsum zurück und verband sie mit dem positiven Einfluss der intestinalen Mikroben.

Ein Probiotikum wird laut World Health Organization (WHO) wie folgt definiert: "Lebende Mikroorganismen, die, wenn sie in ausreichender Menge aufgenommen werden, dem Wirtsorganismus einen gesundheitlichen Nutzen bringen.» Für die Herstellung von probiotischen Therapien werden diverse Bakterienstämme wie Laktobazillen, Enterokokken oder Escherichia coli verwendet. $\mathrm{Zu}$ den Probiotika werden pragmatisch auch Hefepräparate, z.B. Saccharomyces boulardii, gerechnet, obwohl Hefepilze weder zur natürlichen Darmflora gehören noch permanent unseren Darm besiedeln. Tabelle 1 listet die wichtigsten in der Schweiz erhältlichen Probiotika auf.

Die Wirksamkeit der probiotischen Therapie bei CED wurde in mehreren klinischen Studien belegt. Die bahnbrechende randomisierte kontrollierte Studie zum Einsatz des Stamms E. coli Nissle wurde 1999 in Lancet publiziert [1]. Der Effekt eines

Tab. 1. In der Schweiz erhältliche Probiotika (Auswahl)

\begin{tabular}{ll}
\hline Bakterienart & Präparate \\
\hline Milchsäurebakterien & Lactoferment \\
& BactoSan FOS \\
& Symbiolact, Lactibiane \\
& VSL\# 3 \\
& Gynoflor, Infloral \\
\hline Enterokokken & Bioflorin \\
& Symbioflor 1 \\
\hline Coli-Bakterien & Mutaflor \\
& Symbioflor 2 \\
& Colibiogen \\
\hline Hefepilze & Perenterol \\
\hline
\end{tabular}

Probiotikums wurde mit einer Standardtherapie mit Mesalazin verglichen und erwies sich sowohl bei der Remissionsinduktion als auch bei der Remissionserhaltung als ebenbürtig und fast nebenwirkungslos. Die Wirksamkeit der Probiotika bei CED wurde in weiteren klinischen Studien bestätigt.

Gemäss der Erfahrungen aus der Komplementärmedizin erfolgt die probiotische Therapie bei CED, wie bei anderen chronischen Leiden, langfristig in mehreren Stufen:

- Vorphase: Präparate abgetöteter Mikroorganismen (Antigene); Dauer ca. 2 Monate.

- Phase 1: Präparate lebender Enterokokken und Laktobazillen; Dauer ca. 2 Monate.

- Phase 2: Präparate lebender E. coli; Dauer ca. 2 Monate.

- Phase 3: Einsatz der individuell hergestellten Autovakzine (oral und parenteral) aus den eigenen Darmflorakeimen; Dauer ca. 2-4 Monate.

Neben dem Einsatz bei CED existiert für Probiotika eine ausreichende Evidenz für weitere Indikationen wie Durchfall, Obstipation oder Prävention urogenitaler Infektionen (Tab. 2).

Als Vorbereitung für eine langfristige probiotische Therapie wird in der ganzheitlichen Behandlung sinnvollerweise eine Serie von Colon-HydroTherapien durchgeführt. Bei dieser, bereits seit mehreren Jahrzehnten bekannten naturheilkundlichen Methode handelt es sich um eine Darmspülung in einem geschlossenen System (Darmbad). Eine Colon-Hydro-Therapie übt in erster Linie eine entlasten-

\section{KARGER}

Fax +497614520714 Information@Karger.con www.karger.com (c) 2013 S. Karger GmbH, Freiburg
Dr. med. Cesar Winnicki

Chefarzt Aeskulap-Klinik

Gersauerstrasse 8, 6440 Brunnen, Schweiz

Cesar.Winnicki@aeskulap.com 
Tab. 2. Indikationen für eine probiotische Therapie

Modulation des Immunsystems

Reduktion von Diarrhöen (Rotaviren; assoziiert mit Antibiotika; Clostridium difficile, E. coliEnterotoxine)

Behandlung der CED (Colitis ulcerosa, Morbus Crohn)

Reduktion von Helicobacter pylori

Laktoseintoleranz

Prävention urogenitaler Infektionen

Obstipation

de und entgiftende Wirkung besonders auf den Dickdarm aus, führt jedoch auf lange Sicht zu einer vegetativen Umstimmung der Darmfunktion. Unter Berücksichtigung der Indikationen und Kontraindikationen - und technisch sachgemäss durchgeführt - bringt die Colon-HydroTherapie keine Risiken mit sich.

Die standardmässige, konventionelle Therapie von CED besteht in einer reversiblen Suppression der Entzündungsprozesse, sei es durch Mesalazin, Kortikoide oder Zytostatika. Das Ziel der komplementären Therapie in Bezug auf das Immunsystem ist die Modulation und Ausbalancierung der gestörten Abwehrlage. In diesem Sinne stehen uns folgende Massnahmen zur Verfügung: Behandlung mit Thymusextrakten, Ozon-Sauerstoff-Therapie sowie Behandlung mit Wurmeiern (Schweinepeitschenwurm (Trichuris suis ova, TSO)).

Die Thymusdrüse gehört zu den zentralen Immunorganen, die für die Reifung und Schulung der T-Lymphozyten und somit der gesamten zellulären Abwehr zuständig sind. Die höchste Aktivität der Thymusdrüse fällt auf das Kindesalter, wohingegen deren Involution in der Pubertät einsetzt - mit einer allmählichen, aber fast vollständigen Rückbildung. Die bovinen Thymuspeptide werden aufgrund ihrer Ähnlichkeit mit humanen Peptiden sowie der guten Verträglichkeit bereits seit über 50 Jahren medizinisch angewandt. Die verschiedenen
Thymusprodukte haben gemeinsam, dass sie im Knochenmark besonders die Lymphopoese anregen und durch die Verbesserung des Verhältnisses zwischen Helfer- und Suppressorzellen stabilisierend auf das periphere Immunsystem wirken. Die klassischen Indikationen für die Behandlung mit Thymusextrakten sind chronische Infektionen und Autoimmunprozesse. Die meisten Thymuspräparate werden parenteral einbis mehrmals pro Woche über mehrere Monate verabreicht [2].

Die intravenöse Ozonbehandlung gehört zu den klassischen Massnahmen der ärztlichen Komplementärmedizin und beruht auf der ausgeprägten oxidativen Wirkung des dreiatomigen Sauerstoffmoleküls, des Ozons. Etwa $200 \mathrm{ml}$ Eigenblut des Patienten werden in einem geschlossenen System mit einer SauerstoffOzon-Mischung vermengt. Beide Gase werden vollständig durch das venöse Blut absorbiert und dem Patienten retransfundiert. Die Menge des Ozons variiert je nach Indikation zwischen 1 und $5 \mathrm{mg}$ pro Behandlung. Intrakorporal kommt es zur Spaltung des dritten Sauerstoffatoms mit einer akuten oxidativen Wirkung. Der induzierte oxidative Stress führt zur langfristigen Aktivierung diverser antioxidativer Systeme - mit positiver Wirkung auf die Mikrozirkulation, die Regenerationsprozesse sowie das Immunsystem [3]. Bei korrekter Durchführung und Beachtung der Kontraindikationen ist die SauerstoffOzon-Behandlung sicher und nebenwirkungsfrei. Die Therapie wird anfänglich mehrmals pro Woche, später in grösseren Abständen (z.B. einmal pro Monat) wiederholt durchgeführt.

Durch mehrere epidemiologische Studien wurde nachgewiesen, dass der Kontakt mit gewissen Mikroorganismen unserer Umgebung die Funktion des Immunsystems positiv und nachhaltig beeinflusst. In diesem Zusammenhang ist der Einfluss von Parasitenbefall als besonders starke
Immunstimulanz bekannt. In den subtropischen und tropischen Ländern, in denen Parasitenerkrankungen zum Alltag gehören, sind die CED-Erkrankungen äusserst selten [4]. Basierend auf dieser Beobachtung wurde eine neue Behandlungsoption für CED entwickelt, die sich die immunomodulative Wirkung der Parasiten zunutze macht. Als Therapeutikum werden die Eier des TSO verwendet, da dieser Wurm den menschlichen Verdauungstrakt lediglich passager infestiert und eine risikofreie Behandlungsmöglichkeit bietet. Die ersten, im Jahr 1999 publizierten Erfahrungen mit TSO sorgten für ein gewisses Aufsehen bei den Gastroenterologen - ein beachtlich grosser Prozentsatz therapieresistenter Colitis-ulcerosaPatienten erreichte unter dieser Therapie eine Vollremission [5]. Seither wurden einige randomisierte kontrollierte Studien mit dem TSO durchgeführt, die zwar eine positive Wirkung bestätigten, jedoch leider bei einem weit kleineren Teil der Betroffenen. Das Standardprotokoll der Behandlung mit TSO besteht aus einer oralen Verabreichung von 2500 Parasiteneiern alle 2 Wochen. Das fertige TSOPräparat kann offiziell beim Hersteller in den USA und in Deutschland bezogen werden. Aufgrund der hohen Kosten und der schwierigen Logistik hat diese sinnvolle Therapieoption bis anhin nur einen geringgradigen Bekanntheitsgrad erreicht.

Die Phytotherapie bietet ein vielfältiges Spektrum an sowohl symptomatischen als auch ursächlichen Therapiemöglichkeiten bei CED. Die typischen gastrointestinalen Beschwerden von CED-Patienten werden durch pflanzliche Amara-Stoffe, Choleretika, Hepatoprotektiva oder Spasmolytika gelindert. Zu den wichtigsten infrage kommenden Heilkräutern gehören: Kamille (Matricaria chamomilla), Bittere Schleifenblume (Iberis amara), Kümmel (Carum carvi), Schöllkraut (Chelidonium majus) oder Mariendistel (Carduus maria- 
nus). Je nach Beschwerdebild und Verlauf werden diese Heilkräuter individuell entweder als Magistralrezeptur oder Fertigpräparate verschrieben. Neben diesem symptomatischen Einsatz von Heilkräutern gilt ein besonderes Interesse den Phytotherapeutika, die eine direkte Beziehung zu CED-Erkrankungen aufweisen und deren Wirksamkeit in klinischen Studien belegt wurde. Der Weihrauch (Boswellia serrata) ist für seine antientzündliche Wirkung vor allem im Sinne der Blockierung von 5-Lipoxygenase (5-LOX) bekannt. In einer randomisierten Studie bei CED-Patienten war die Wirksamkeit von Weihrauch mit Mesalazin vergleichbar [6]. Dass Ballaststoffe eine positive Wirkung auf den Verdauungstrakt inklusive einer Reduktion der Krebsinzidenz aufweisen, ist bereits lange bekannt. Die Schalen von Indischen Flohsamen (Plantago ovata) werden gerne als Quellstoff gegen Obstipation eingesetzt. Darüber hinaus unterstützt dieser pflanzliche Ballaststoff die Remissionserhaltung bei Colitis ulcerosa, und zwar mit der Standardmedikation (Mesalazin) vergleichbar [7]. Eine weitere Option in der Behandlung von chronisch-entzündlichen Erkrankungen stellt die Gelbwurz (Curcuma longa) dar. Diese im Fernen Osten wohlbekannte und verbreitete Gewürzpflanze enthält eine Reihe von hochwirksamen, antientzündlichen und kanzeroprotektiven Stoffen wie z.B. Curcumin. Obwohl das Haupteinsatzgebiet für Gelbwurz in der Behandlung von Prostataleiden liegt, wird C. longa aufgrund ihres interessanten Wirkspektrums (antientzündlich, choleretisch) auch bei CED eingesetzt [8].

Mikronährstoffe im Sinne der orthomolekularen Therapie bieten eine weitere wichtige Option im ganzheitlichen Management von CED-Patienten. Die Bedeutung von Vitamin D3 und Calcium bei der Prävention der Osteoporose ist allgemein bekannt. Hingegen wird die Versorgung mit Zink und Selen im Allgemeinen weniger beachtet [9]. Die aktuelle Versorgung mit diesen essenziellen Spurenelementen kann mittels Vollblutanalyse (hohe intrazelluläre Konzentration) beurteilt werden. Bei einem Mangel ist eine langfristige Substitution angezeigt, die sich positiv sowohl auf das Immunsystem als auch auf die antioxidativen Systeme auswirkt. Die ungesättigten Fettsäuren, insbesondere die Omega-3-Fettsäuren, spielen in unserem Körper eine entscheidende Rolle bei der Regulation entzündlicher Prozesse [10]. Obwohl für Omega-3-Fettsäuren kein direkter Einfluss auf die CED-Erkrankungen nachgewiesen werden konnte, ist deren Einsatz aus systemischer Sicht sinnvoll und klinisch relevant. Im Arsenal der orthomolekularen Behandlung befindet sich auch die Aminosäure Glutamin, die besonders in Stresssituationen die bevorzugte Energiequelle für Gehirnzellen, Leukozyten, aber auch Enterozyten darstellt [11].

Morbus Crohn und Colitis ulcerosa gelten als klassische Beispiele für psychosomatische Erkrankungen. Sowohl charakteristische Persönlichkeits- oder Reaktionsmuster als auch eine Abhängigkeit von Stresssituationen, von Jahreszeiten und vom sozialen Umfeld wurden beschrieben. Aus diesen Gründen ist eine individuelle und konsequente psychotherapeutische Betreuung für die langfristige Prognose dieser Patienten entscheidend. In klinischen Studien konnte beispielsweise die positive Wirkung der medizinischen Hypnose bei Patienten mit CED belegt werden [12]. An dieser Stelle darf im Sinne der komplementärmedizinischen Behandlung wohl kaum die Klassische Homöopathie fehlen. Obwohl vonseiten der konventionellen Medizin nach wie vor weder verstanden noch akzeptiert, spielt die individuelle konstitutionelle Homöopathie oft eine erfolgskritische Rolle.
Fallbeispiel für eine erfolgreiche integrative Behandlung von CED

Als der 12-jährige Marcus wegen seiner Colitis ulcerosa im Februar 1997 stationär in die Aeskulap-Klinik aufgenommen wurde, hatte er bereits eine 6-jährige, turbulente Vorgeschichte hinter sich. Die Diagnose Colitis ulcerosa wurde im Jahr 1991 während seiner ersten Hospitalisation im Kinderspital Luzern gestellt; im März 1993 musste er aufgrund einer weiteren Exazerbation erneut 4 Wochen stationär behandelt werden. Anschliessend kam es unter Dauerbehandlung mit Kortison und Azathioprin allmählich zur Stabilisierung des Verlaufs. Eine akute VarizellenErkrankung mit Fieber und systemischer Manifestation (Lunge, Bewusstsein) im Juni 1996 hatte den dritten Spitalaufenthalt zur Folge. Die eingeleitete antivirale Therapie verursachte einen massiven Leberschaden, sodass man sowohl die antivirale als auch die immunosuppressive Behandlung einstellen musste. Nach der Entlassung war der gesundheitliche Zustand von Marcus leidlich kompensiert, und ein nächster Schub der CED liess nicht lange auf sich warten. Im Dezember 1996 traten wieder blutige Durchfälle auf und der allgemeine Zustand des Jungen verschlechterte sich vor allem wegen einer Anämie zusehends. Nach langer Auseinandersetzung mit der zuständigen Krankenversicherung wurde der Junge schlussendlich in die Aeskulap-Klinik hospitalisiert. Aus dem Bereich der konventionellen Medizin wurde vorsichtshalber lediglich Mesalazin oral verabreicht. Die aufgedeckten Mängel an Selen und Zink wurden parenteral substituiert und eine langfristige, mehrphasige probiotische Therapie wurde eingeleitet. Im Sinne der Immunmodulation erhielt der Knabe regelmässig intravenöse Ozon-Sauerstoff-Therapien. Das Konzept wurde durch Klassische Homöopathie, Phyto- und Psychotherapie vervollständigt. 
Nach 5 Wochen konnte Marcus in stabilem Allgemeinzustand und mit akzeptabler gastrointestinaler Funktion nach Hause entlassen werden. Die integrative Behandlung wurde konsequent ambulant fortgesetzt. Im Sommer 1997 (ca. 3 Monate nach dem Beginn der integrativen Behandlung) normalisierte sich die Funktion des Verdauungstrakts vollständig. Ende 1998 wurden Mesalazin und Ende 1999 die Probiotika abgesetzt, ohne dass es jemals zu einer weiteren Exazerbation kam. Die letzte offizielle Konsultation erfolgte wegen Acne vulgaris im Jahr 2003; die Darmfunktion war zu diesem Zeitpunkt nach wie vor normal. Mittlerweile beträgt die gesamte Follow-up-Zeit 15 Jahre; es wurden keine Colitis-spezifischen Beschwerden beobachtet.

\section{Literatur}

1 Remibacken BJ, et al: Non-pathogenic Escherichia coli versus mesalazine for the treatment of ulcerative colitis: a randomised trial. Lancet 1999;354:635-639.

2 Wilson JL: Thymus Extracts: An International Literature Review of Clinical Studies. Foundation for Immunology and Nutrition, Development, Education and Research 1999; $1-25$.

3 Bocci VA: Scientific and medical aspects of ozone therapy. State of the art. Arch Med Res 2006;37:425-435.

4 Falcone FH, Pritchard DI: Parasite role reversal: worm on trial. Trends Parasitol 2005;21:157-160.

5 Summers RW, et al: Trichuris suis therapy for active ulcerative colitis: a randomized controlled trial. Gastroenterology 2005;128: 825-832.
6 Gupta I, et al: Effects of Boswellia serrata gum resin in patients with ulcerative colitis. Eur J Med Res 1997;2:37-43.

7 Fernández-Bañares F, et al: Randomized clinical trial of Plantago ovata seeds (dietary fiber) as compared with mesalamine in maintaining remission in ulcerative colitis. Am J Gastroenterol 1999;94:427-433.

8 Jurenka JS: Anti-inflammatory properties of curcumin, a major constituent of Curcuma longa: a review of preclinical and clinical research. Altern Med Rev 2009;14: 141-153.

>9 Rath HC, et al: Mangelzustände und Komplikationen bei chronisch entzündlichen Darmerkrankungen. Med Klin 1998;93:610.

10 Yashodhara BM, et al: Omega-3 fatty acids: a comprehensive review of their role in health and disease. Postgrad Med J 2009;85:84-90.

11 Novak F, et al: Glutamine supplementation in serious illness: a systematic review of the evidence. Crit Care Med 2002;30:2022-2029.

12 Schmidt CF: Hypnotic suggestions and imaginations in the treatment of colitis ulcerosa. Hypnos 1992;19:237-242. 